\title{
Drug resistance among infantile enteropathogenic Escherichia coli strains isolated in the United Kingdom
}

\author{
R J GROSS， LINDA R WARD， E J THRELFALL， HILARY KING，B ROWE
}

\begin{abstract}
Two hundred and thirty-two strains of Escherichia coli belonging to infantile enteropathogenic serotypes isolated in the United Kingdom during 1980 and 1981 were tested for resistance to 10 antimicrobial drugs. Resistance to one or more drugs was found in $134(57.8 \%)$ of the strains, with resistance to sulphonamides, streptomycin, tetracycline, and ampicillin occurring most commonly. Resistance was transferable in 65 out of 104 resistant strains.

These findings are a cause for concern because they indicate that the choice of treatment for severe illness is limited and suggest that a large pool of drug-resistant organisms exists in the community.
\end{abstract}

\section{Introduction}

Severe outbreaks of infantile enteritis due to enteropathogenic Escherichia coli have occurred in Europe and the United States. Such outbreaks, however, have rarely been reported during the past 10 years: sporadic cases continue to occur, but it is often difficult to assess the importance of enteropathogenic $E$ coli isolated from the stools. The increasing problem of multiple transferable drug resistance among Salmonella and Shigella has been well documented recently, but statistics for $E$ coli are not available. ${ }^{1}$ As part of a continuous surveillance of drug resistance in the Enterobacteriaceae we have studied a sample of strains of enteropathogenic $E$ coli isolated in the United Kingdom.

\section{Methods}

Bacterial strains-We examined 232 strains of $E$ coli.isolated in the United Kingdom during 1980-1 from the faeces of infants under 3 years old. The patients were epidemiologically unrelated; where outbreaks had occurred only one isolate from each outbreak was included. One hundred and sixty-nine infants had diarrhoea while 24 did not have diarrhoea; in 39 cases this information was not received. The strains belonged to recognised enteropathogenic $E$ coli serogroups: 018ac (34 strains), 026 (28), 044 (7), 055 (18), 086 (16), $0111(20)$, 0114 (15), 0119 (8), 0125 (13), 0126 (15), 0127 (13), 0128 (41), and $0142(4)$.

Drug resistance tests-Initial screening for resistance to ampicillin (A), chloramphenicol $(\mathrm{C})$, gentamicin $(\mathrm{G})$, neomycin/kanamycin $(\mathrm{K})$, streptomycin (S), and tetracyclines $(\mathrm{T})$ was performed by a diffusion method. ${ }^{2}$ Strains found to be resistant by this method were further tested by an agar dilution method. ${ }^{3}$ Resistance to sulphonamides $(\mathrm{Su})$, trimethoprim ( $\mathrm{Tm}$ ), furazolidone (Fu), and nalidixic acid $(\mathrm{Nx})$ was also tested by the agar dilution method. The drug concentrations used in the agar dilution tests are shown in the table.

Transfer and mobilisation of drug resistance-One hundred and four resistant strains were tested for transfer of drug resistance using a

\footnotetext{
Division of Enteric Pathogens, Central Public Health Laboratory, London NW9 5HT

R J GROSS, MA, MSC, principal microbiologist LINDA R WARD, BSC, principal microbiologist E J THRELFALL, BSC, PHD, principal microbiologist HILARY KING, medical laboratory scientific officer B ROWE, MB, FRCPATH, director
}

nalidixic-acid-resistant strain of $E$ coli $\mathrm{K} 12, \mathrm{~F}^{-} 1 \mathrm{ac}^{+}$(strain No $14 \mathrm{R} 525)$, as the recipient. ${ }^{4}$ If no direct transfer was detected mobilisation of resistance was attempted using autotransferring plasmids of several different compatibility groups. ${ }^{5}$

Characterisation of plasmids-Autotransferring and non-autotransferring resistance plasmids were characterised by the methods of Anderson and Threlfall ${ }^{2}$ and tested for their incompatibility with suitable representatives of the plasmid compatibility groups listed by Willshaw et al. ${ }^{6}$

Drug resistance of 232 strains of infantile enteropathogenic $E$ coli isolated from faeces of infants in the $U K$

\begin{tabular}{lcc}
\hline Drug & $\begin{array}{c}\text { Concentration } \\
\text { of drug (mg/l) }\end{array}$ & $\begin{array}{c}\text { No (\%) of strains } \\
\text { resistant }\end{array}$ \\
\hline Ampicillin & 8 & $86(37 \cdot 1)^{*}$ \\
Chloramphenicol & 8 & $29(12 \cdot 5) \dagger$ \\
Furazolidone & 20 & $1(0 \cdot 4)$ \\
Gentamicin & 4 & 0 \\
Nalidixic acid & 20 & 0 \\
Neomycin & 8 & $21(9 \cdot 1)$ \\
Streptomycin & 16 & $106(45 \cdot 7)$ \\
Sulphonamides & 100 & $106(45 \cdot 7)$ \\
Tetracycline & 16 & $67(28 \cdot 9)$ \\
Trimethoprim & $0 \cdot 5$ & $14(6 \cdot 0)$ \\
\hline No $(\%)$ fully sensitive & & $98(42 \cdot 2)$ \\
\hline
\end{tabular}

*All these strains were also resistant at $256 \mathrm{mg} / 1$

$\dagger$ Twenty-eight of these strains were also resistant at $256 \mathrm{mg} / \mathrm{l}$

\section{Results}

Resistance to sulphonamides, streptomycin, tetracycline, and ampicillin occurred most commonly (table). One hundred and thirty-four strains $(57.8 \%)$ were resistant to one or more drugs : 22 strains $(9.5 \%)$ were resistant to one drug, $27(11.6 \%)$ to two, and $85(36.6 \%)$ to three or more. Twenty-nine strains $(12.5 \%)$ were resistant to chloramphenicol, and all of these were resistant to at least three other drugs.

Of the 104 resistant strains tested for transfer of resistance, 65 $(62.5 \%)$ were able to transfer resistance directly and resistance was mobilised in a further six $(5 \cdot 8 \%)$. Plasmids were characterised in 40 of the 65 strains in which direct transfer of resistance was observed and in six strains with mobilisable resistance. Five strains carried two autotransferring resistance plasmids and four carried additional transfer factors that coded for the production of colicin Ib but not for drug resistance. Plasmids of the $\mathrm{B}$ and $\mathrm{F}$ compatibility groups predominated. Group B plasmids were identified in strains from nine serogroups, and the most common resistance pattern transferred was ASSu (22 strains). In contrast, plasmids of the F group coded for up to six resistances, the most common pattern being ACKSSuT. Non-autotransferring but mobilisable resistances were encoded by transfer-defective plasmids of the $\mathrm{H}_{2}$ and $\mathrm{F}_{\mathrm{II}}$ compatibility groups and by $\mathrm{SSu}$ and $\mathrm{A}$ resistance determinants.

\section{Discussion}

Most infants with $E$ coli diarrhoea recover uneventfully if water and electrolyte disturbances are corrected promptly, and antimicrobial drug treatment is rarely necessary. Infants with severe illness, however, have sometimes been reported to respond dramatically to antibiotic treatment, and such treatment should certainly be considered for prolonged or severe illnesses. Antibiotics have also been used in attempting to prevent the spread of infection, although the evidence for their value is equivocal.

The present study shows that about $60 \%$ of enteropathogenic 
$E$ coli strains isolated in the United Kingdom are drug resistant and that resistance is transferable in more than $60 \%$ of these. The majority of autotransferring resistance plasmids identified were of the B and F compatibility groups. Group B plasmids are particularly common in shigellae and have been observed in strains of Shigella dysenteriae 1 that have caused epidemics in Central America ${ }^{8}$ and Asia. ${ }^{9}$ F-like resistance plasmids are common in salmonellae, and $E$ coli and plasmids of the $\mathrm{F}_{\mathrm{I}}$ me group have been observed in salmonella strains that have caused outbreaks of gastroenteritis in the Middle East, ${ }^{10}$ India, ${ }^{11}$ and southern Europe. ${ }^{12}$

These findings are a cause for concern not simply because they indicate that the choice of treatment for severe illnesses is limited but also because they suggest the existence of a large pool of drug-resistant organisms in the community. Multiple drug resistance may be transferred in vivo to other pathogenic organisms including those $E$ coli responsible for systemic infections such as neonatal meningitis. Further studies are in progress to determine the prevalence of drug resistance in $E$ coli strains isolated from cerebrospinal fluid.

\section{References}

1 Rowe B, Threlfall EJ. Multiple antimicrobial resistance in enteric pathogens. F Antimicrob Chemother $1981 ; 7: 1-3$.
2 Anderson ES, Threlfall EJ. The characterisation of plasmids in the enterobacteria. 7 Hyg 1974; 72:471-87.

${ }^{3}$ Haltalin KC, Markley AH, Woodman E. Agar plate dilution method for routine antibiotic susceptibility testing in a hospital laboratory. $A m \mathcal{F}$ Clin Pathol 1973;60:384-94.

4 Anderson ES, Lewis MJ. Characterisation of a transfer factor associated with drug resistance in Salmonella typhimurium. Nature 1965;208: 843-9.

${ }^{5}$ Anderson ES. A rapid screening test for transfer factors in drug-sensitive Enterobacteriaceae. Nature 1965;208:1016-7.

6 Willshaw GA, Threlfall EJ, Ward LR, Ashley AS, Rowe B. Plasmid studies of drug-resistant epidemic strains of Salmonella typhimurium belonging to phage types 204 and 193. F Antimicrob Chemother 1980;6:763-73.

7 Lambert HP. Antimicrobial agents in diarrhoeal disease. Clin Gastroenterol $1979 ; 8: 827-33$

${ }^{8}$ Grindley NDF, Grindley JN, Anderson ES. R factor compatibility groups. Molecular and General Genetics 1972;119:287-97.

${ }^{9}$ Frost JA, Rowe B, Vandepitte J, Threlfall EJ. Plasmid characterisation in the investigation of an epidemic caused by multiply resistant Shigella dysenteriae type 1 in Central Africa. Lancet 1981 ;ii:1074-6.

10 Anderson ES, Threlfall EJ, Carr JM, McConnell MM, Smith HR. Clonal distribution of resistance plasmid-carrying Salmonella typhimurium, mainly in the Middle East. $f$ Hyg 1977;79:425-48.

11 Rowe B, Frost JA, Threlfall EJ, Ward LR. Spread of a multiresistant clone of Salmonella typhimurium phage type 66/122 in South East Asia and the Middle East. Lancet 1980; :1070-1.

${ }^{12}$ McConnell MM, Smith HR, Leonardopoulos J, Anderson ES. The value of plasmid studies in the epidemiology of infections due to drugresistant Salmonella wien. 7 Infect Dis 1979;139:178-89.

(Accepted 29 fune 1982)

\title{
Reuse of haemodialysis equipment: convenience and cost effectiveness
}

\author{
H K BANESTER， R D DRISCOLL， R N GREENWOOD， W R CATTELL
}

\begin{abstract}
The shortage of resources for providing renal replacement makes it essential to reduce revenue costs wherever possible. Assuming that haemodialysis is likely to remain a mainstay of renal replacement, a policy of reusing the expensive disposable dialysers and blood lines could offer substantial cost savings. Such reuse has been shown to be safe and to maintain efficiency, but it must also take account of convenience to the patient, especially those dialysing at home. A survey of patients treated with home dialysis shows that the rate of reuse of the complete extracorporeal circuit was highest for those patients who had automated reuse systems.
\end{abstract}

\section{Introduction}

The treatment of patients with end-stage renal failure by haemodialysis has been highly successful. Nevertheless,

\footnotetext{
Department of Nephrology, St Bartholomew's and St Leonard's Hospitals, London

H K BANESTER, HND, assistant administrator R D DRISCOLL, BA, MLITT, home administrator R N GREENWOOD, MSC, MRCP, research registra W R CATTELL, MD, FRCP, consultant physician
}

haemodialysis is expensive and several hundred patients die each year in the United Kingdom because the financial resources to provide treatment are lacking. ${ }^{12}$ In the United States, where there has been a quite lavish allocation of federal resources for dialysis programmes, there is increasing pressure to contain or reduce costs. ${ }^{34}$ At the same time most units in the United Kingdom and elsewhere are moving away from the use of the bulky Kiil-type artificial kidneys towards the use of small disposable dialysers, which require less work from staff and patients and take up less space in the home. This means, however, that the dialyser and other disposable equipment become a major revenue cost.

Though considerable savings could be made by reusing these disposable items, this policy has, however, met with resistance from both clinicians and patients on the grounds of safety, efficiency, and convenience. The safety and efficiency of the procedure is now widely accepted both in Europe and in the USA, ${ }^{5}{ }^{6}$ but convenience to, and acceptance by, patients has received little attention. Since most patients in the United Kingdom dialyse at home, the success of any policy aimed at more economic use of materials depends on their co-operation. A recent survey of home dialysis practice in our unit has given us the opportunity to examine the extent to which our own policy of reuse is implemented in the home.

\section{Cost benefits of reuse}

Single use of a disposable dialyser is expensive, about $£ 1900$ a year. Reuse (in our case to a total of six times) dramatically reduces this cost by more than half (figure). It is less commonly recognised that the arterial and venous blood lines, which-with the dialyser-form the extracorporeal circuit, are also expensive. If the dialyser can be reused 